\title{
Controlling the Resonance of a Photonic Crystal Microcavity by a Near-Field Probe
}

\author{
A. Femius Koenderink, ${ }^{1, *}$ Maria Kafesaki, ${ }^{2}$ Ben C. Buchler, ${ }^{1}$ and Vahid Sandoghdar ${ }^{1, \dagger}$ \\ ${ }^{1}$ Laboratory of Physical Chemistry, Swiss Federal Institute of Technology (ETH), 8093 Zürich, Switzerland \\ ${ }^{2}$ Institute of Electronic Structure and Laser (IESL), Foundation for Research and Technology Hellas (FORTH), P.O. Box 1527, \\ 71110 Heraklion, Crete, Greece
}

(Received 15 December 2004; published 5 October 2005)

\begin{abstract}
We demonstrate theoretically that the resonance frequencies of high- $Q$ microcavities in twodimensional photonic crystal membranes can be tuned over a wide range by introducing a subwavelength dielectric tip into the cavity mode. Three-dimensional finite-difference time-domain simulations show that by varying the lateral and vertical positions of the tip, it is possible to tune the resonator frequency without lowering the quality factor. Excellent agreement with a perturbative theory is obtained, showing that the tuning range is limited by the ratio of the cavity mode volume to the effective polarizability of the nanoperturber.
\end{abstract}

DOI: 10.1103/PhysRevLett.95.153904

Solid-state optical microresonators are of great interest to a wide range of fields such as biosensing, nonlinear optics, low-threshold lasers, and cavity quantum electrodynamics $[1,2]$. Two cavity properties that are commonly desired, but often incompatible, are very high quality factors $(Q)$ and very small mode volumes. Photonic crystal microcavities in thin semiconductor membranes [3-5] promise a good compromise between these features. One of the difficulties associated with such monolithic ultrasmall resonators is to match their resonance frequencies with those of interest in a given application. This is especially a concern since fabrication tolerances make it nearly impossible to realize the exact design parameters. Temperature tuning has been used to meet a resonance condition, as was recently shown in the case of coupling between a quantum dot and a microcavity [6]. In fact, this was possible due to the temperature sensitivity of the quantum dot. In general, however, it is desirable to independently tune the cavity resonance without manipulating the system that it couples to. In this Letter we show that the introduction of a subwavelength dielectric object, such as a scanning probe, can achieve this.

A scanning near-field optical microscope (SNOM) tip has recently been used to image the intensity distribution in a low- $Q$ photonic crystal microresonator [7]. The central idea in our current work is to investigate how the presence of an external dielectric nanometer-sized object, such as a SNOM tip, can modify the resonance condition of a photonic crystal microresonator [see Fig. 1(a)]. This scheme is analogous to a technique common in microwave engineering whereby the frequency of a resonator is adjusted by insertion of dielectric stubs [8]. In that case, because microwave resonators are typically closed, scattering from the object does not cause any loss, and the cavity $Q$ is determined by absorption. However, when a dielectric object is placed into the field of an open cavity, such as a photonic crystal slab resonator, scattering dominates. Indeed, it has been shown experimentally that coupling of a glass fiber tip can shift the resonance frequency of a
PACS numbers: 42.70.Qs, 42.50.Pq, 42.60.Da

high- $Q$ microresonator at the cost of reducing its quality factor [9]. Since a photonic crystal microcavity has a very small mode volume and relies on the precise arrangement of dielectric material at the subwavelength scale, one might expect that introducing the slightest external object would spoil the quality factor [10]. In what follows we show that this is not necessarily the case. It turns out that a small dielectric object acts in the same fashion as an atom which, within classical linear dispersion theory, changes the frequency of a high-finesse cavity according to its polarizability and its position relative to the nodes of the cavity mode $[11,12]$.

We consider a dielectric cylindrical tip near a membrane-type photonic crystal, consisting of a high index slab that is perforated with a hexagonal lattice (lattice constant $a=420 \mathrm{~nm}$ ) and surrounded by air. We assume a slab dielectric constant $\epsilon=11.76$, a thickness of $250 \mathrm{~nm}$, and a hole radius $r=0.3 a$. As Fig. 1(a) shows, the resonator is formed about a defect of reduced radius $r=0.15 a$, similar to the optimization in Ref. [3]. By reducing the radius of just two holes to $r=0.23 a$ on either side of the defect and then shifting them outwards by $0.11 a$, we create a nondegenerate dipole mode with a mode profile as shown in Fig. 1(b). The mode has a frequency $\omega a / 2 \pi c=0.284$ in the center of the 2D band gap. The $Q$ is about 13000 , corresponding to a resonance linewidth of $15 \mathrm{GHz}$ at $\lambda=$ $1500 \mathrm{~nm}$. Crystals over $5 \times 5 \mu \mathrm{m}^{2}$ in lateral size surrounded by up to $1 \mu \mathrm{m}$ of air were simulated using the 3D finite-difference time-domain (FDTD) method with Liao's absorbing boundary conditions [13-15]. Computational meshes were 14 or 20 grid points per $a$ parallel to the membrane and had doubled resolution normal to the membrane [16]. Grid-cell volume averaging of the dielectric constant was employed to reduce staircasing errors [17]. Quality factors and cavity mode frequencies were obtained by fitting a damped harmonic wave to time traces of the total $E$-field energy in the cavity.

Figure 1(c) displays a contour plot of the relative frequency shift $\Delta \omega / \omega$ as a function of the lateral position of a 

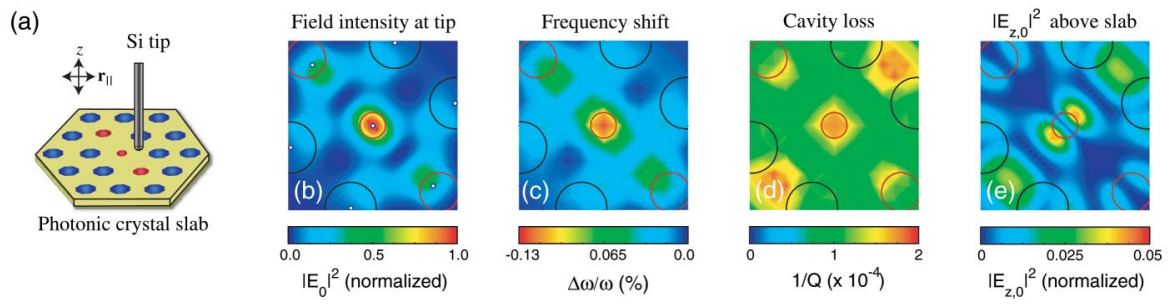

FIG. 1 (color). (a) Schematic of the system under study. A cylindrical silicon tip with a diameter of $125 \mathrm{~nm}$ is placed at height $z_{\text {tip }}$ and lateral position $\mathbf{r}_{\|}$above a photonic crystal slab. Reduction of diameters for 3 pores (shaded red) and shift of two of them yields a cavity resonance at $\lambda=1500 \mathrm{~nm}$ with a $Q$ factor of 13000 . (b) Intensity distribution $\left|\mathbf{E}_{0}\right|^{2}$ of the unperturbed mode in a plane $30 \mathrm{~nm}$ above the slab. The black circles outline the air pores (red circles for tuned pores), whereas the white dots mark the original pore locations in the hexagonal lattice. (c) Calculated resonance frequency shift induced by the tip placed $30 \mathrm{~nm}$ above the slab, as a function of its lateral position. (d) The inverse $Q$ corresponding to (c). The unperturbed cavity has $1 / Q=7.5 \times 10^{-5}$. (e) Contribution of the $z$ component $\left|E_{z, 0}\right|^{2}$ to the unperturbed field intensity, integrated over the extent of the tip above the slab. Field intensities in (b) and (e) are normalized to the maximum total field intensity $\left|\mathbf{E}_{0}\right|^{2}$.

silicon tip of $125 \mathrm{~nm}$ diameter, placed at a height of $30 \mathrm{~nm}$ above the photonic crystal slab. As expected, the introduction of high index material in the cavity mode causes a redshift of the mode frequency, depending on the tip position. The maximum relative frequency shift of $0.13 \%$ amounts to about $2 \mathrm{~nm}$ (or $260 \mathrm{GHz}$ ) at $\lambda=1500 \mathrm{~nm}$ and occurs when the tip is placed above the central defect. At a lateral distance of $200 \mathrm{~nm}$ from the central hole, the shift is already less than $10 \%$ of this maximum value, but as the tip approaches the two shifted holes, again a large tuning of up to $200 \mathrm{GHz}$ can be achieved. As recently shown by Yoshie et al. [6], such frequency shifts of many cavity linewidths open the possibility of controlled quantum optical experiments. Figure 2 displays the dependence of the cavity resonance on the separation between the tip apex and the photonic crystal slab $\left(z_{\text {tip }}\right)$, for the case where the tip is aligned with the cavity center. The filled circles show that as the tip is approached from afar $\left(z_{\text {tip }}>0\right)$, the frequency shift grows exponentially with a $1 / e$ length of $d=50 \mathrm{~nm}$. When the tip is put through the slab $\left(z_{\text {tip }}<0\right)$, the increasing amount of dielectric material inserted in the mode profile continuously detunes the resonance to the red, saturating at a shift of $\Delta \omega / \omega=-3.7 \%$. The black curve in Fig. 2 displays the variation of the unperturbed cavity mode intensity $\left|\mathbf{E}_{0}\right|^{2}$. The excellent agreement between this curve and the filled symbols for $z_{\text {tip }}>0$ indicates that the frequency shift maps $\left|\mathbf{E}_{0}\right|^{2}$. This direct correspondence of the frequency shift to the mode profile is also evident from the strong similarity of the lateral images in Figs. 1(b) and 1(c).

An exciting aspect of cavity tuning with a tip is that a large frequency shift can be achieved without inducing a considerable cavity loss. Open symbols in Fig. 2 show that within the accuracy of the simulations the cavity $Q$ of 13000 has not been affected at separations down to about $60 \mathrm{~nm}$ while the cavity resonance has been tuned by $100 \mathrm{GHz}$. Even at a height of $30 \mathrm{~nm}$ where $\Delta \omega=$ $260 \mathrm{GHz}$, the cavity $Q$ remains as high as 5000 . If the tip is pushed closer to or into the central defect hole, however, the continuous increase of the frequency shift is accompa- nied by a strong reduction in $Q$. In this regime, the tip perturbs the mode profile and strong out-of-plane scattering occurs. When the tip extends completely through the defect slab and the distribution of dielectric material becomes more symmetric, the $Q$ recovers to about 1000 .

To gain quantitative insight into the modification of the cavity resonance, we have taken advantage of the established theory of dielectric perturbations in microwave cavities $[8,18]$. Suppose the tip has a dielectric constant of $\epsilon_{p}$ and occupies the volume $V_{p}$. Maxwell's relations imply the exact expression,

$$
\begin{aligned}
\frac{\Delta \omega}{\omega} & -\frac{i}{2 \Delta Q} \\
& =\frac{-\int_{V_{p}}\left[\epsilon_{p}-\epsilon(\mathbf{r})\right] \operatorname{Re}\left[\mathbf{E}_{0}^{*} \cdot \mathbf{E}_{p}\right] d \mathbf{r}+\frac{i}{\omega} \oint_{\delta V} \operatorname{Re}\left[\mathbf{S}_{p}\right] \cdot \mathbf{n} d a}{\int_{V} \operatorname{Re}\left[\mathbf{E}_{0}^{*} \cdot \mathbf{D}_{p}+\mathbf{H}_{0}^{*} \cdot \mathbf{B}_{p}\right] d \mathbf{r}}
\end{aligned}
$$

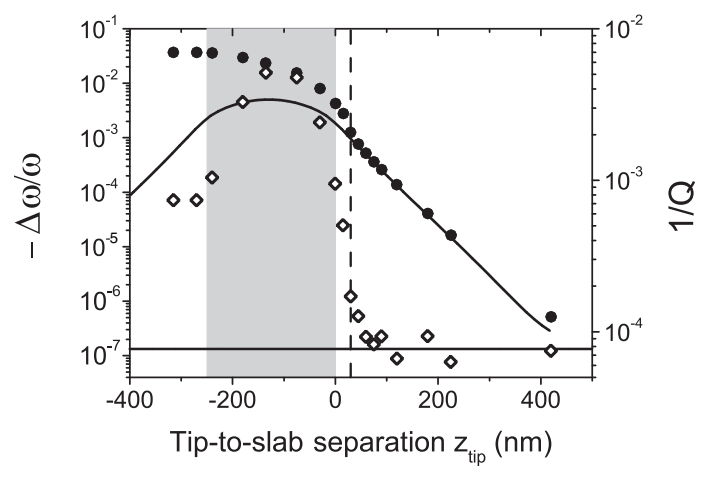

FIG. 2. Relative frequency shift (solid symbols, left hand axis) and inverse $Q$ (open symbols, right hand axis) as a function of the distance $z_{\text {tip }}$ between the surface of the photonic crystal and a Si tip of $125 \mathrm{~nm}$ diameter aligned with the central defect. The gray area indicates the extent of the membrane. For $z_{\text {tip }}<0$ the tip extends into the defect. The dashed line indicates $z_{\text {tip }}=$ $30 \mathrm{~nm}$ corresponding to the data in Fig. 1. The black curve (scaled) shows the unperturbed mode profile $\left|\mathbf{E}_{0}\right|^{2}$. The horizontal line indicates $1 / Q$ in absence of the tip. 
for the relative frequency shift $(\Delta \omega / \omega)$ and induced scattering loss $\left(\Delta Q^{-1}\right)$. Here $\epsilon(\mathbf{r})$ is the dielectric constant of the unperturbed system and $\mathbf{E}_{0(p)}$, and $\mathbf{H}_{0(p)}$ are the complex electric and magnetic fields of the unperturbed (perturbed) system. The integral in the denominator runs over some volume $V$ enclosing the entire system. The induced scattering loss is proportional to the integrated flux of $\mathbf{S}_{p}=$ $\mathbf{E}_{p}^{*} \times \mathbf{H}_{0}-\mathbf{E}_{0}^{*} \times \mathbf{H}_{p}$ through the outer surface $\delta V$ of $V$. We note that this dependence is very different from that of the frequency shift that is proportional to an integral over the perturbing volume $V_{p}$.

A simple picture for the frequency shift emerges in the weakly perturbative regime where $\frac{\Delta \omega}{\omega} \ll 1$ and the $Q$ is not degraded, in our case corresponding to the range $z_{\text {tip }} \geq$ $30 \mathrm{~nm}$. In this regime, the denominator in Eq. (1) is independent of the perturbation, and the frequency shift is set by the overlap integral of the perturbed and unperturbed fields within the tip volume $V_{p}$ only. The field $\mathbf{E}_{p}$ in the tip is given by $\mathbf{E}_{p}=3 \mathbf{E}_{0} /\left(\epsilon_{p}+2\right)$, where the proportionality constant takes into account the local field effects [18]. Next, we separate the exponential $z$ dependence of $\mathbf{E}_{0}=$ $\mathbf{E}_{0}\left(\mathbf{r}_{\|}\right) e^{-z / 2 d}$ from its dependence on the lateral tip coordinate $\mathbf{r}_{\|}$, and assume that the unperturbed field $\mathbf{E}_{0}\left(\mathbf{r}_{\|}\right)$is constant over the small tip cross section. We then find

$$
\frac{\Delta \omega\left(\mathbf{r}_{\|}, z_{\text {tip }}\right)}{\omega}=-\frac{\alpha_{\text {eff }}}{2 V_{\text {cav }}} \frac{\left|E_{0}\left(\mathbf{r}_{\|}\right)\right|^{2}}{\max \left[\epsilon(\mathbf{r})\left|E_{0}\right|^{2}\right]} e^{-z_{\text {tip }} / d} .
$$

Here $V_{\text {cav }}=\int \epsilon(\mathbf{r})\left|E_{0}\right|^{2} d \mathbf{r} / \max \left[\epsilon(\mathbf{r})\left|E_{0}\right|^{2}\right]$ is defined as the cavity mode volume [3,18] and $\alpha_{\text {eff }}$ is the effective polarizability of the tip

$$
\alpha_{\mathrm{eff}}=3 \frac{\epsilon_{p}-1}{\epsilon_{p}+2} V_{\mathrm{eff}}
$$

equal to the electrostatic polarizability of a volume $V_{\text {eff }}$ of material with dielectric constant $\epsilon_{p}$ [19]. The exponential decay of the cavity mode (with decay length $d$ ) limits the volume of the tip that contributes to the polarizability to $V_{\text {eff }}=\pi r_{p}^{2} d$ for a tip of radius $r_{p}$ [20]. This analysis reproduces all the features of our FDTD results concerning the frequency shift. First, it yields the exponential decrease of $\Delta \omega / \omega$ as a function of the tip-slab separation $z_{\text {tip }}$ (see Fig. 2). Second, it confirms the direct correspondence between the frequency shift and the unperturbed mode profile [see Figs. 1(b) and 1(c)]. Third, Eq. (2) states that the frequency shift is inversely proportional to the mode volume, a feature that we have confirmed by simulations of different cavity designs (e.g., that of Ref. [4]). Further confirmation is the quantitative agreement of the frequency shifts in Fig. 2 with the mode volume $V_{\text {cav }}=0.02 \mu \mathrm{m}^{3}$ determined from the unperturbed mode profile of our cavity. Finally, and most significantly, Eq. (2) predicts that the frequency shift is proportional to $\alpha_{\text {eff }}$. To investigate this we performed FDTD simulations for tips of various radii and materials, fixed above the cavity center at $30 \mathrm{~nm}$. The closed symbols and the solid line in Fig. 3 demonstrate that the frequency shift is proportional to the effective polarizability of the tip. In other words, in the region $z_{\text {tip }}>30 \mathrm{~nm}$ the tip acts as a polarizable object that is weakly coupled to the cavity field, much in the same manner as an atom that can be described by a classical dipole of the appropriate polarizability within linear dispersion theory $[11,12]$. This is a remarkable finding because the tip is not much smaller than the central hole of the microcavity and its mode volume, so that the validity of the dipole approximation is not a trivial matter.

Next we turn our attention to the origin of losses induced by the tip. The power radiated by an object of polarizability $\alpha_{\text {eff }}$ can be modeled as a Rayleigh scattering process $[19,21]$, resulting in a quadratic dependence on $\alpha_{\text {eff }}$. Indeed, the open symbols in Fig. 3 display exactly this dependence when the tip is at the center of the cavity. The quadratic dependence of the loss versus the linear dependence of the frequency shift on $\alpha_{\text {eff }}$ makes it possible to tune the frequency of high- $Q$ microcavities without incurring prohibitive losses. Both perturbative analysis [21] as well as FDTD simulations indicate that the robustness of tip tuning against loss generalizes to other cavities. For the cavities in Refs. [4,6], for instance, we again find second order dependence of loss on frequency shift, with a large shift of $\Delta \omega / \omega=0.1 \%$ at a high $Q=4500$. Intriguing effects beyond this Rayleigh scattering picture, however, occur for some tip configurations. For instance, a comparison of Figs. 1(b) and 1(d) shows that there are tip positions where the losses are high even though the electric field intensity is small (upper right and lower left corners). It turns out, as shown in Fig. 1(e), that the cavity field at these points has a large $z$ component $\left(E_{z, 0}\right)$. The magnitude of $\left|E_{z, 0}\right|^{2}$ at these points amounts to about $5 \%$ of the maxi-

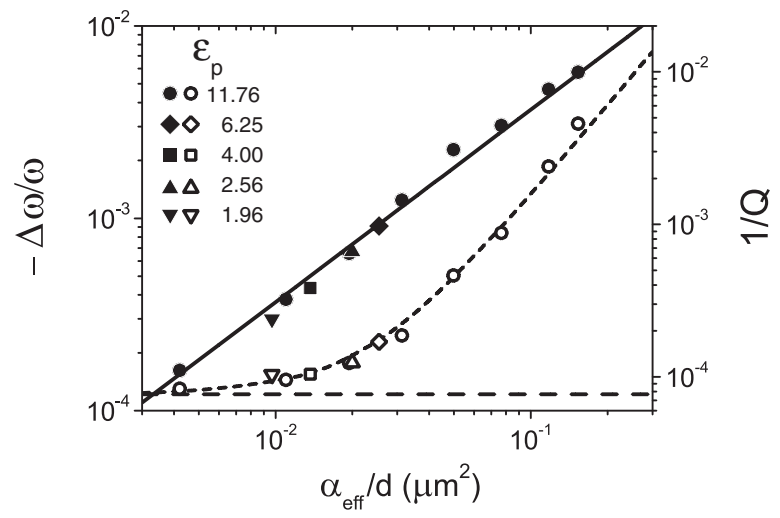

FIG. 3. Relative frequency shift (solid symbols, left hand axis) and inverse $Q$ (open symbols, right hand axis) as a function of the effective polarizability per length $\alpha_{\text {eff }} / d=3\left(\epsilon_{\mathrm{p}}-1\right) /\left(\epsilon_{\mathrm{p}}+\right.$ 2) $\pi r_{\mathrm{p}}^{2}$, for cylindrical tips positioned $30 \mathrm{~nm}$ above the central defect in Fig. 1. Symbol shapes indicate various tip materials ( $\mathrm{Si}$, $\mathrm{TiO}_{2}, \mathrm{ZrO}_{2}$, polystyrene, and $\mathrm{SiO}_{2}$ in order of decreasing $\epsilon_{\mathrm{p}}$ as indicated). The frequency shift is proportional to $\alpha_{\text {eff }}$ (solid curve). The loss $1 / Q$ can be fitted (dashed curve) with the sum of the loss without the tip (horizontal dashed line) and a contribution proportional to $\alpha_{\mathrm{eff}}^{2}$. 
mum value of $\left|\mathbf{E}_{0}\right|^{2}$ attained above the center of the cavity. Substantial loss is induced by scattering of the field into the tip, which is much more efficient for the $z$ component of the field than for the field components perpendicular to the tip [22]. FDTD simulations of the perturbed fields further confirm the polarization-specific enhanced coupling into the tip [23]. This disparity between induced loss and the cavity mode profile underlines a fundamental feature of Eq. (1): contrary to the frequency shift, the scattering loss does not depend solely on the fields within the perturbing volume only. Instead, it is the far-field interference of the fields encoded in the integrated flux $\mathbf{S}_{p}$ that is the decisive factor. In this sense, there is a close relation between the perturbation of a cavity by a tip and the tuning of holes around a cavity for optimization of the quality factor [3-5].

In conclusion, we have shown that sharp dielectric tips in the near field can be used to tune photonic crystal microcavities over a large range without inducing prohibitive losses. Our analysis shows that the tuning range scales with the ratio of the tip polarizability to the mode volume. Hence, this scheme is expected to be effective for any electromagnetic resonance localized in a volume comparable to the perturbing polarizability, as is the case in microspheres, microdisks, or micropillars $[2,6,9]$. One advantage of this scheme is that the tuning process does not influence the optical properties of emitters in the cavity. Furthermore, the rapid progress in nanotechnology makes it feasible to integrate tips for local tuning of individual resonant devices in photonic crystals that contain many functional components [24]. Compared to existing methods for tuning photonic crystals [25], tip tuning can create and reverse the frequency shift more quickly, and, in contrast to ultrafast optical tuning [26], the shift can be maintained indefinitely. In other words, tip tuning does not depend on the material properties of the crystal. The time scale for tuning is limited only by mechanical resonances of the tip that can reach the $\mathrm{MHz}$ regime [24]. This opens the possibility of using a photonic crystal microcavity as an optical switch for integrated optics applications.

This work was funded by the Deutsche Forschungsgemeinschaft (DFG) through focus program SP1113 and by ETH Zürich. M. K. thanks Professor C. M. Soukoulis for continuous support and fruitful discussions and acknowledges support from EU projects DALHM, METAMORPHOSE, and PHOREMOST.

*Present address: FOM Institute for Atomic and Molecular Physics, Kruislaan 407, NL-1098 SJ Amsterdam, The Netherlands.

†Electronic address: vahid.sandoghdar@ethz.ch

[1] Optical Processes in Microcavities, edited by R. K. Chang and A. J. Campillo (World Scientific, Singapore, 1996).

[2] K. J. Vahala, Nature (London) 424, 839 (2003).

[3] J. Vučković, M. Lončar, H. Mabuchi, and A. Scherer, Phys. Rev. E 65, 016608 (2001).
[4] Y. Akahane, T. Asano, B. S. Song, and S. Noda, Nature (London) 425, 944 (2003).

[5] K. Srinivasan, P. E. Barclay, O. Painter, J. X. Chen, A. Y. Cho, and C. Gmachl, Appl. Phys. Lett. 83, 1915 (2003).

[6] T. Yoshie, A. Scherer, J. Hendickson, G. Khitrova, H. M. Gibbs, G. Rupper, C. Ell, O. B. Shchekin, and D. G. Deppe, Nature (London) 432, 200 (2004).

[7] P. Kramper, M. Kafesaki, C. M. Soukoulis, A. Birner, F. Müller, R. Wehrspohn, U. Gösele, J. Mlynek, and V. Sandoghdar, Opt. Lett. 29, 174 (2004).

[8] R. A. Waldron, Proc. Inst. Electr. Eng. 107C, 272 (1960); for a review see O. Klein, S. Donovan, M. Dressel, and G. Grüner, Int. J. Infrared Millim. Waves 14, 2423 (1993).

[9] S. Götzinger, O. Benson, and V. Sandoghdar, Appl. Phys. B 73, 825 (2001).

[10] For an example where this assumption is made, see p. 6 of S. Fan, P.R Villeneuve, J. D. Joannopoulos, and H. A. Haus, Phys. Rev. B 64, 245302 (2001).

[11] Cavity Quantum Electrodynamics, edited by P. R. Berman (Academic, San Diego, 1994), and references therein.

[12] Y. Zhu, D. J. Gauthier, S.E. Morin, Q. Wu, H. J. Carmichael, and T.W. Mossberg, Phys. Rev. Lett. 64, 2499 (1990).

[13] A. Taflove and S.C. Hagness, Computational Electrodynamics: The Finite-Difference Time-Domain Method (Artech House, Boston, MA, 2000), 2nd ed.

[14] S. Fan, I. Appelbaum, and J.D. Joannoupoulos, Appl. Phys. Lett. 75, 3461 (1999).

[15] Simulations for various air layer thicknesses above $0.5 \mu \mathrm{m}$ showed that the boundaries did not affect $\omega$ and $Q$.

[16] M. Kafesaki, M. Agio, and C. M. Soukoulis, J. Opt. Soc. Am. B 19, 2232 (2002).

[17] O. Hess, C. Hermann, and A. Klaedtke, Phys. Status Solidi A 197, 605 (2003).

[18] R. Inoue, H. Kitano, and A. Maeda, J. Appl. Phys. 93, 2736 (2003).

[19] J.D. Jackson, Classical Electrodynamics (John Wiley \& Sons, New York, 1975).

[20] FDTD results show reduced frequency shifts consistent with the reduced volume for tips of limited length. As the tip length increases, the shift saturates exponentially at the value set by $V_{\text {eff }}$, with $1 / e$ length equal to $d$.

[21] M. Kuznetsov and H. A. Haus, IEEE J. Quantum Electron. 19, 1505 (1983); S. C. Hill, H. I. Saleheen, and K. A. Fuller, J. Opt. Soc. Am. A 12, 905 (1995).

[22] C. Girard, A. Dereux, O. J. F. Martin, and M. Devel, Phys. Rev. B 50, 14467 (1994).

[23] See EPAPS Document No. E-PRLTAO-95-051541 for a PDF file containing contour plots (color) of the electric field in cuts normal to the photonic crystal slab studied in the Letter. This document can be reached via a direct link in the online article's HTML reference section or via the EPAPS homepage (http://aip.org/pubservs/epaps.html).

[24] See H. G. Craighead, Science 290, 1532 (2000), and references therein.

[25] B. Maune, M. Lončar, J. Witzens, M. Hochberg, T. BaehrJones, D. Psaltis, A. Scherer, and Y. Qiu, Appl. Phys. Lett. 85, 360 (2004).

[26] S. W. Leonard, H. M. van Driel, J. Schilling, and R. B. Wehrspohn, Phys. Rev. B 66, 161102(R) (2002); P.E. Barclay, K. Srinivasan, and O. Painter, Opt. Express 13, 801 (2005). 